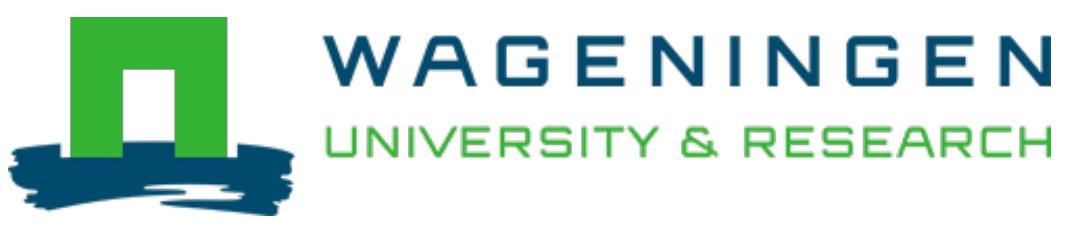

\title{
Development of a multi-position indentation setup : Mapping soft and patternable heterogeneously crosslinked polymer networks
}

\author{
Review of Scientific Instruments \\ Boots, J.N.M.; Fokkink, R.; Gucht, J.; Kodger, T.E. \\ https://doi.org/10.1063/1.5043628
}

This article is made publicly available in the institutional repository of Wageningen University and Research, under the terms of article $25 \mathrm{fa}$ of the Dutch Copyright Act, also known as the Amendment Taverne. This has been done with explicit consent by the author.

Article 25 fa states that the author of a short scientific work funded either wholly or partially by Dutch public funds is entitled to make that work publicly available for no consideration following a reasonable period of time after the work was first published, provided that clear reference is made to the source of the first publication of the work.

This publication is distributed under The Association of Universities in the Netherlands (VSNU) 'Article 25fa implementation' project. In this project research outputs of researchers employed by Dutch Universities that comply with the legal requirements of Article $25 \mathrm{fa}$ of the Dutch Copyright Act are distributed online and free of cost or other barriers in institutional repositories. Research outputs are distributed six months after their first online publication in the original published version and with proper attribution to the source of the original publication.

You are permitted to download and use the publication for personal purposes. All rights remain with the author(s) and / or copyright owner(s) of this work. Any use of the publication or parts of it other than authorised under article $25 \mathrm{fa}$ of the Dutch Copyright act is prohibited. Wageningen University \& Research and the author(s) of this publication shall not be held responsible or liable for any damages resulting from your (re)use of this publication.

For questions regarding the public availability of this article please contact openscience.library@,wur.nl 
Development of a multi-position indentation setup: Mapping soft and patternable heterogeneously crosslinked polymer networks

Cite as: Rev. Sci. Instrum. 90, 015108 (2019); https://doi.org/10.1063/1.5043628

Submitted: 11 June 2018 . Accepted: 19 December 2018. Published Online: 10 January 2019

J. N. M. Boots (D), R. Fokkink, J. van der Gucht (D), and T. E. Kodger (D)
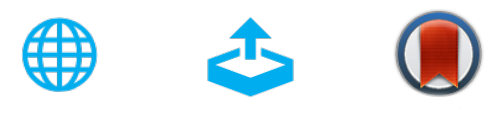

View Online

Export Citation

CrossMark

\section{ARTICLES YOU MAY BE INTERESTED IN}

Temperature gradients for thermophysical and thermochemical property measurements to $3000{ }^{\circ} \mathrm{C}$ for an aerodynamically levitated spheroid

Review of Scientific Instruments 90, 015109 (2019); https://doi.org/10.1063/1.5055738

Visible astro-comb filtered by a passively stabilized Fabry-Perot cavity

Review of Scientific Instruments 90, 013102 (2019); https://doi.org/10.1063/1.5053706

Performance and application of heavy ion nuclear microbeam facility at the Nuclear Physics Institute in Řě̌, Czech Republic

Review of Scientific Instruments 90, 013701 (2019); https://doi.org/10.1063/1.5070121

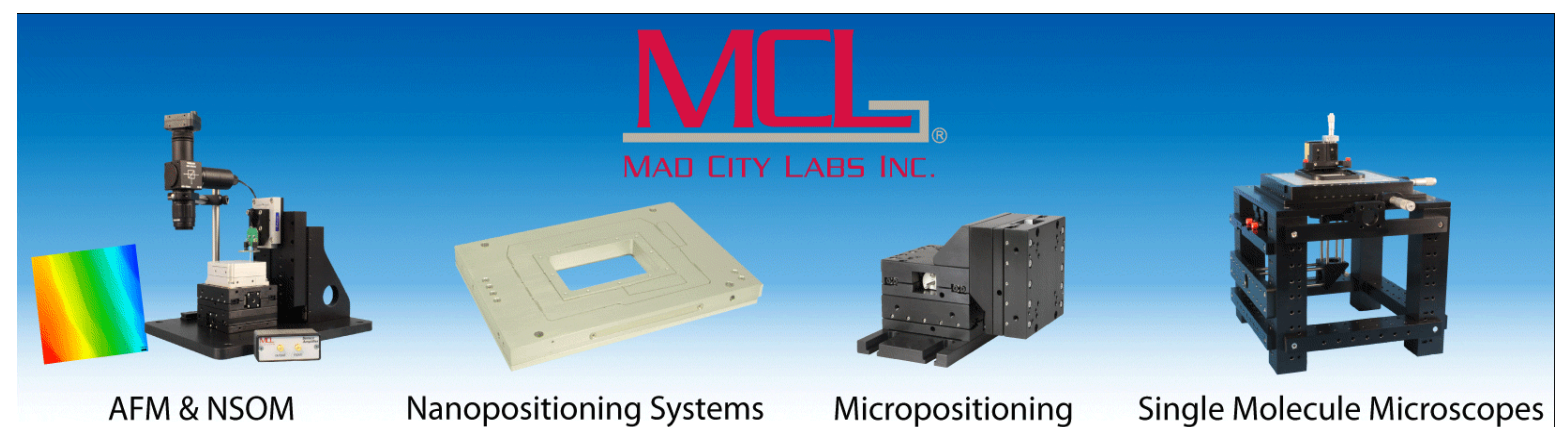




\title{
Development of a multi-position indentation setup: Mapping soft and patternable heterogeneously crosslinked polymer networks
}

Cite as: Rev. Sci. Instrum. 90, 015108 (2019); doi: 10.1063/1.5043628

Submitted: 11 June 2018 - Accepted: 19 December 2018 •

Published Online: 10 January 2019

J. N. M. Boots, (D) R. Fokkink, J. van der Gucht, (iD and T. E. Kodger ${ }^{a)}$ (iD)

\section{AFFILIATIONS}

Physical Chemistry and Soft Matter, Wageningen University and Research, Stippeneng 4, 6708WE Wageningen, The Netherlands

a) Author to whom correspondence should be addressed: thomas.kodger@wur.nl

\begin{abstract}
We present the development of a multi-position indentation setup capable of spatially mapping mechanically heterogeneous materials. A detailed description of the indentation instrumentation is first provided, emphasizing force sensitivity, noise reduction, and signal fidelity. We first present indentation experiments on soft hydrogels that are submerged in water and show how the large contributions to the measured force due to the air-water surface tension can be avoided. The displacement field of the indented hydrogel is visualized using fluorescently coated microspheres embedded in the hydrogel, allowing simultaneous mapping of the stress and strain fields for a soft polymer network. We then fabricate a polymer network with patterned elasticity using halftone UV lithography and map the elastic modulus with the multi-position indentation instrument. The applied UV pattern is found back in the measured elastic modulus map, showing the capability of the multi-position indentation setup to map mechanically heterogeneous polymer networks.
\end{abstract}

Published under license by AIP Publishing. https://doi.org/10.1063/1.5043628

\section{INTRODUCTION}

Polymeric materials have become indispensable to everyday life due to their widespread applications. The properties of polymer materials are determined by their molecular structure and connectivity. Contrary to crystalline materials, polymers have an intrinsically disordered structure, which is characterized by heterogeneities that are frozen during preparation of the materials. The amplitude and typical length scale of these heterogeneities can be particularly large for hydrogels, i.e., polymer networks swollen in water, where coupling between the cross-linking reaction and the local monomer density can lead to large spatial variations in crosslink density, especially when the gel is close to the critical gel point. ${ }^{1-5}$ Biological hydrogel systems such as collagen and fibrin biopolymer networks also contain mechanical heterogeneities on larger length scales due to local variations in polymer density. ${ }^{6,7}$ These heterogeneities have a large effect on the mechanical response of the materials, as they lead to non-affine deformations ${ }^{8}$ and stress localization in regions where cracks may eventually nucleate. 9,10 More recently, several groups have tried to exploit heterogeneity to design materials with a desired mechanical response. ${ }^{1-15}$

It is clear from these examples that there is a need for characterization methods that can probe the properties of the material locally. Examples of conventional methods to probe the mechanical properties of polymer materials include rheology or Dynamic Mechanical Analysis (DMA), which measure average properties of a specimen, ${ }^{16,17}$ and Atomic Force Microscopy (AFM), which effectively probes the surface of a specimen and not the bulk and practically cannot probe regions that are larger than a hundred square micrometers. ${ }^{6,18,19}$ A technique that can be used to measure mechanical heterogeneities on larger length scales $(\mu \mathrm{m} \mathrm{cm})$ is indentation, in which a spherical probe is pushed against the sample and the resulting normal force is measured. ${ }^{20,21}$ By performing indentations at multiple positions, a spatially resolved elasticity map can be obtained. However, to fully characterize the 
mechanics in a heterogeneous soft material, information on the distribution of stresses within the material is crucial. This requires measurement of the strain field in the material under indentation and calls for the incorporation of imaging possibilities. To realize this, we present a spatially high-precision and mechanically sensitive multi-position indentation setup, which, due to its flexible design, can be easily integrated with imaging capabilities. We show how this combination can be used to independently determine the local Young's modulus and Poisson ratio of the material. In addition, we describe how the method can be used to characterize materials submerged in a fluid, where the effects of buoyancy and capillary forces need to be accounted for. The structure of this article is as follows: first, we discuss the principles of the indentation experiment and a detailed description of the instrument; second, we show data on the indentation of submerged hydrogels; third, we show the combination of indentation with fluorescence microscopy to measure the displacement field in hydrogels using fluorescently labeled microspheres; and lastly, we show mechanical mapping of a micro-patterned polydimethylsiloxane (PDMS) network that directly demonstrates the benefit of the built multi-position indentation instrument.

\section{CONTACT MECHANICS}

A mechanical test that is suited to probe mechanical properties of a material locally is indentation. In an indentation experiment, a probe connected to a load cell is lowered onto a sample, and the resulting normal force is measured, as shown in Fig. 1. The load cell measures a local mechanical response of the material over an area that is determined by the size of the indenting sphere. Hertzian contact theory relates the indentation force between a rigid sphere and a semi-infinite elastic substrate to the mechanical properties of that substrate,

$$
\mathrm{F}=\frac{4}{3} \mathrm{R}^{1 / 2} \delta^{3 / 2} \frac{\mathrm{E}}{1-v^{2}},
$$

where $\mathrm{F}$ is the measured (normal) force, $\delta$ is the indentation depth, $R$ is the radius of the probe, $E$ is the elastic modulus, and $v$ is Poisson's ratio of the elastic body. Here we use a stainless steel probe with an elastic modulus that is orders of magnitude larger than that of the measured polymer samples. The work presented in this article is performed solely with spherical probes, avoiding the mandatory alignment of the probe with the surface of the substrate that is necessary for other configurations such as conical, cylindrical, and flat probes. ${ }^{37}$ Note that in the presence of adhesive (van der Waals) forces between the probe and the sample one should consider the adhesive contact theory developed by Johnson, Kendall, and Roberts, commonly known as JKR theory. ${ }^{22}$ Additionally, corrections for finite samplethickness may be necessary. As shown elsewhere, ${ }^{23}$ these corrections depend on the ratio $\sqrt{\mathrm{R} \delta} / h$ with $h$ being the thickness of the sample. Here, we have ensured that in all measurements $\sqrt{R \delta} / h<0.1$, for which these corrections are negligibly small.

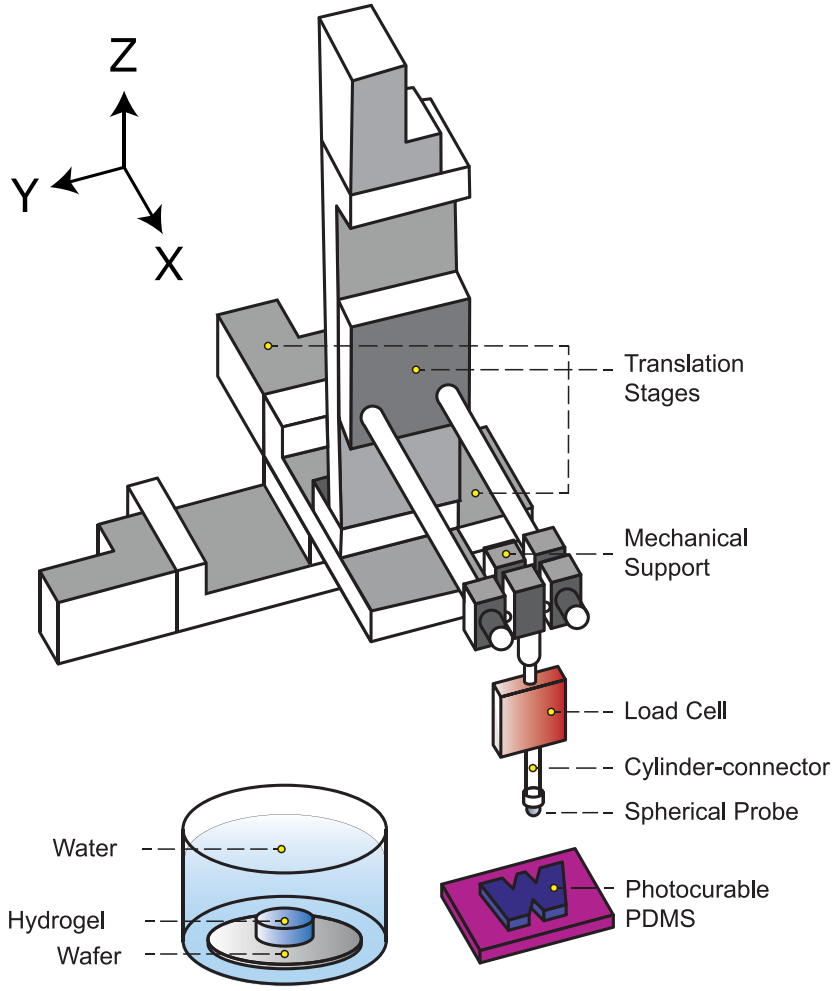

FIG. 1. 3D schematic representation of the experimental instrument. Three translation stages are mounted on top of one another to enable movement of the load cell (red) and the attached probe in three dimensions. Mechanical support is achieved with right-angle clamps (Thorlabs RA90/M), which ensures an orthogonal angle with the sample surface by eliminating rotational degrees of freedom. Two samples are tested: photo-patterned PDMS and hydrogels submersed in water, placed on a silicon wafer that was glued to the bottom of a beaker.

\section{THE MULTI-POSITION SPHERICAL INDENTATION INSTRUMENT}

Indenting a $2 \mathrm{D}$ polymeric substrate at multiple positions requires a force sensor that can be moved not only in the vertical $(\hat{z})$ direction but also in the lateral $(\hat{\mathrm{x}}-\hat{\mathrm{y}})$ directions. To achieve this, three translation stages (M-403.6DG, Physical Instruments), with a minimal incremental movement of $0.2 \mu \mathrm{m}$ and a total travel range of $15 \mathrm{~cm}$, are used to move the load cell in three directions, as shown in Fig. 1. Each translation stage is controlled by communicating to a control box (C-663.12 Mercury Step Stepper Motor Controller, 1 Axis) with Matlab. ${ }^{24}$ The movement of the translation stages and readout of the load cell are performed stepwise.

We use FUTEK load sensors (FSH02667, 20 g limit, nonsubmersible or QSH00618, $100 \mathrm{~g}$ limit, submersible) that are connected to an analog amplifier (SG-3016, ICP DAS) which communicates with the computer via a digital acquisition device (USB-6001, National Instruments); see Fig. 2(a). An RCfilter is used to filter out high frequency electrical noise, as shown in Fig. 2(b). The load cell is sampled every millisecond, 
(a)

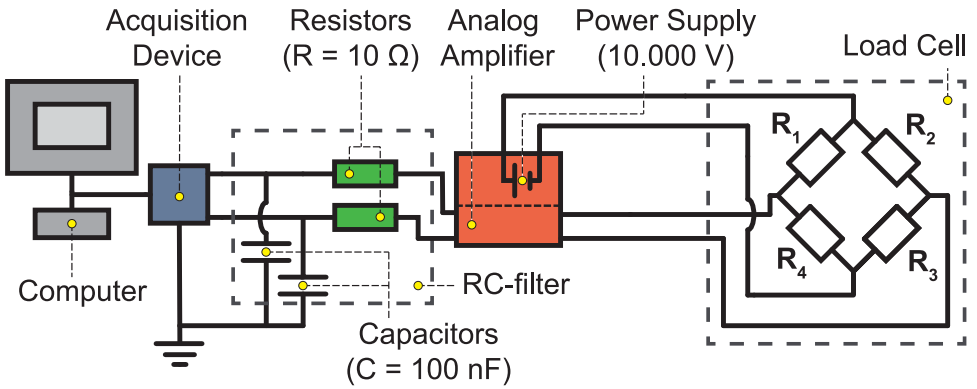

(b)

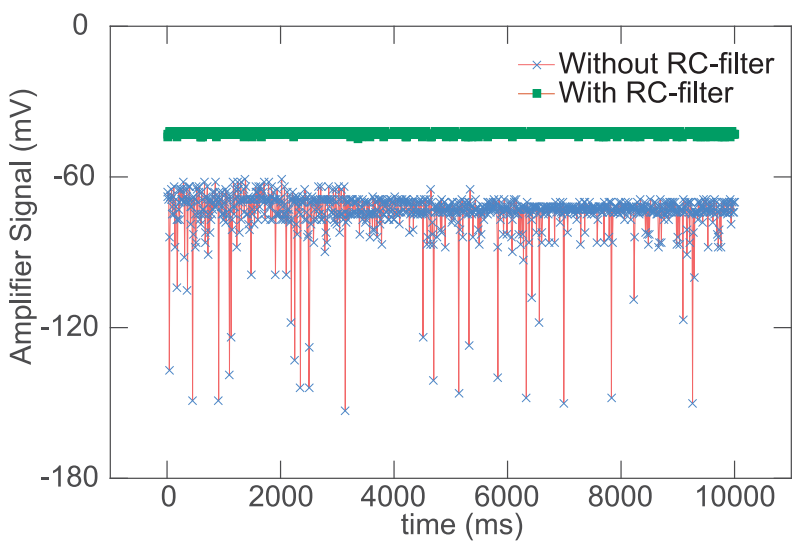

and an average is taken over a thousand samplings. The filtered voltage signal is then converted to the measured force, after calibration with standard weights.

\section{INDENTATION OF SUBMERGED HYDROGELS}

We first test our setup by measuring the elasticity of soft hydrogels. Hydrogels contain large amounts, up to 99.9 mass\%, of water, ${ }^{25}$ resulting in low elastic moduli, especially when the crosslink density is close to the critical gel point. To measure these low elastic moduli, a sensitive load cell is required. However, indentation of a hydrogel in air leads to a contribution in the measured force from the air-water surface tension, $\gamma_{\mathrm{LG}}$, which is approximately $70 \mathrm{mN} / \mathrm{m}$. Since hydrogels contain mostly water by mass, their surface tension is similar to this, ${ }^{26}$ which means that for soft hydrogels the capillary force will dominate the elastic restoring force. Therefore, the hydrogels must be submerged in water while measuring the normal force. Unfortunately, the most sensitive commercially available load cells cannot be submersed into a solvent. To obtain maximal force resolution, we first perform indentation experiments on soft hydrogels submersed in water with a highly sensitive commercially available non-submersible load cell. This means that the cylindrical connector between the load cell and the probe (see Fig. 1) traverses the air/water interface, which results in a capillary contribution to the measured force due to the meniscus of the air-water interface at the surface of the cylindrical connector. Moreover, it means that the buoyancy force changes when the height of the part of the cylinder that is submerged in water changes.
FIG. 2. (a) A schematic of the electrical circuit. The load cell is powered by a stable voltage of $10.000 \mathrm{~V}$ supplied by using the analog amplifier. Thermo-sensitive strain gauges in the Wheatstone bridge are placed oppositely perpendicular and parallel to the tension and compression axes of the load cell, where the latter will respond to an applied load. The stable input voltage is compared to the change in voltage due to compression of the two parallel oriented resistors. A voltage output is returned to the amplifier and passed onto the differential input of a data acquisition device $\left(f_{\max }=10\right.$ $\mathrm{kHz}$ ) through an $\mathrm{RC}$-filter to filter out high frequency noise $\left(\mathrm{R}=10 \Omega\right.$ and $\mathrm{C}=100 \mathrm{nF}, f_{c}=159 \mathrm{kHz}$ ). (b) The voltage measured with and without the RC-filter; the former is offset $+30 \mathrm{mV}$ for visualization purposes.
Upon indenting a submerged hydrogel sample, a forcedistance curve is obtained, as shown in Fig. 3. The raw data are obtained with the more sensitive 20 g-limit non-submersible load cell and require corrections for the capillary force and for the buoyancy force.

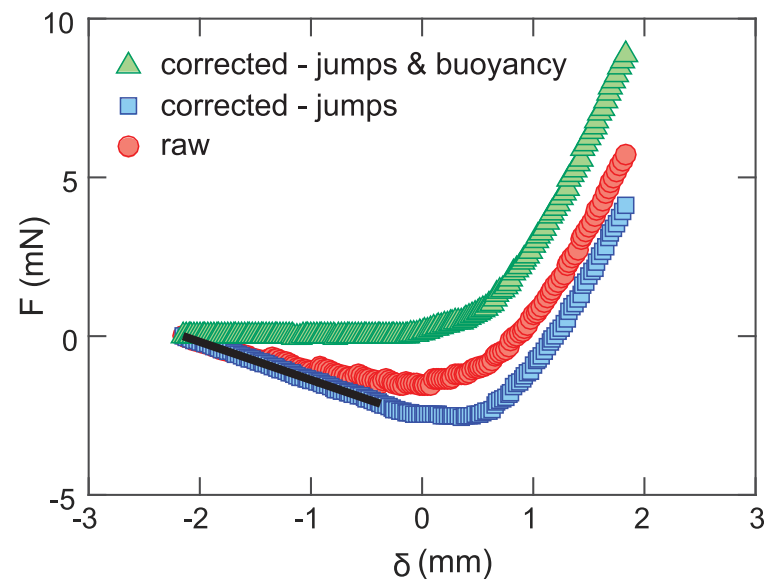

FIG. 3. Indentation of a $10 \mathrm{w} / \mathrm{v} \%$ poly(acrylamide) gel, crosslinked with $1.0 \mathrm{w} / \mathrm{w} \%$ $N, N^{\prime}$-methylenebisacrylamide, measured with a non-submersible load cell and with a probe of radius $R=2 \mathrm{~mm}$. Shown is a single force versus distance curve, i.e., no repeats, for raw data (red circles); data corrected for jumps in the force (blue squares); data corrected for buoyancy, where the black line indicates a fit of the data before contact used for the correction (green triangles). 
For a perfectly smooth surface characterized by an equilibrium contact angle $\theta$, the capillary force $F_{c}$ adds a constant contribution to the measured force, given by

$$
\mathrm{F}_{\mathrm{c}}=\gamma_{\mathrm{LG}} \ell \cos \theta,
$$

where $\ell$ is the circumference of the cylindrical connector. In practice, however, roughness or chemical heterogeneity of the surface leads to contact angle hysteresis and contact line pinning. ${ }^{27}$ This is evident from Figs. 4(a) and 4(c), where the position of the air-water interface and the contact angle at the cylinder surface are shown as a function of time during a single indentation cycle. Clearly the contact angle is not (a)

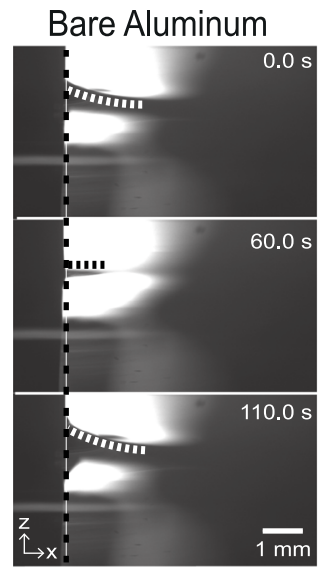

(c)

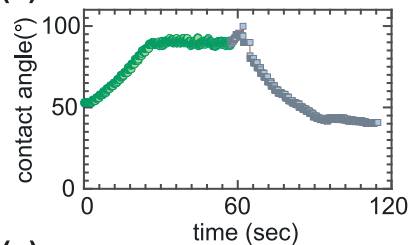

(e)

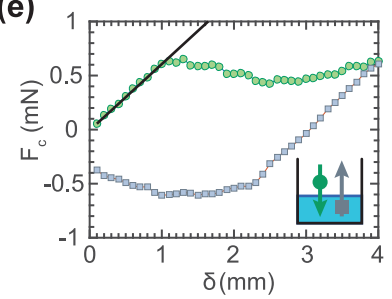

(b)

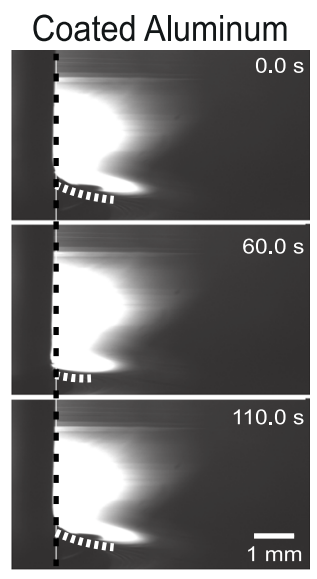

(d)

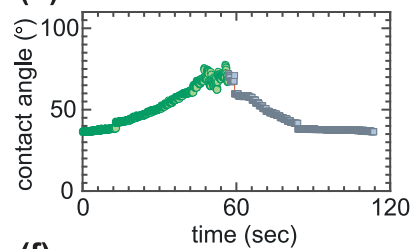

(f)

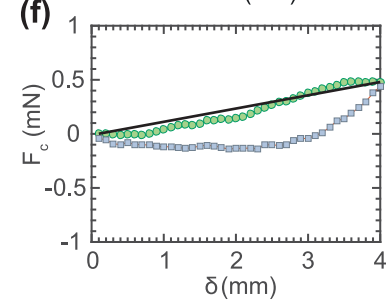

FIG. 4. ( $\hat{x}, \hat{z})$-snapshots of the air-liquid interface and the surface of the cylinderconnector for (a) bare aluminum and (b) aluminum coated with complex coacervates (phase contrast images). In between snapshots, the cylinder-connector was moved $4 \mathrm{~mm}$ down and up again at a velocity of $1 \mathrm{~mm} / \mathrm{s}$, with 80 force-samplings each of $1 \mathrm{~s}$ and $\sim 0.5 \mathrm{~s}$ processing time for a total of $120 \mathrm{~s}$. Snapshots are taken under a small $\sim 20^{\circ}$ angle. The dotted white/black lines indicate the pinning of the air/water interface at the cylinder-connector. The contact angles in (c) and (d) are obtained by selecting an intensity threshold for each frame at the indicated vertical lines. The force-distance curves in (e) and (f) show the capillary force measured by the non-submerged load cell in the absence of a hydrogel, after correcting for buoyancy according to Eq. (3). constant during indentation so that also the capillary force varies in time [Fig. 4(e)]. The intermittent movement of the contact line due to repeated pinning and unpinning at the cylinder surface then leads to jumps in the measured force curve. Indeed, the jumps observed in the raw force curves shown in Fig. 3 have the same magnitude as those observed in Fig. 4(e).

One solution to reduce the magnitude of the jumps in the measured normal force is to eliminate surface irregularities that cause the changes in contact angle. We have attempted to do this by coating the cylinder-connector with a complex coacervate through a sequential layer by layer application of poly(diallyldimethylammonium chloride) and poly(styrenesulfonate) in a $1 \mathrm{M} \mathrm{NaCl}$ solution, increasing the hydrophilicity. As shown in Figs. 4(b) and 4(d), this indeed reduces the contact angle hysteresis and the resulting variations in the capillary force [Fig. 4(f)]. However, even with this coating, contact angle hysteresis is still present so that the jumps in the force curve cannot be eliminated completely. This means that we must correct for the jumps in the force by shifting the measured normal force accordingly after each jump, which is performed here by taking the value of the point prior to the jump and adding the difference between the two preceding points, $F_{n}=F_{n-1}+\left(F_{n-1}-\right.$ $F_{n-2}$ ), where $n$ is the index of the data point that must be corrected.

The second correction that we must apply to the data in Fig. 3 is for the buoyancy force, which arises when an object moves through a fluid thereby displacing the fluid. This force, $F_{b}$, is dependent on the height of the cylindrical connector in the fluid with respect to the fluid's surface, ${ }^{28}$

$$
\mathrm{F}_{b}=-\rho_{\mathrm{F}} \mathrm{gA} \Delta h
$$

in which $\rho_{F}$ is the density of the fluid, $g$ is the gravitational acceleration, A is the cross-sectional area of the cylinder, and $\Delta h$ is the total height of the part of the cylinder that is submersed in the fluid. The latter increases linearly as the probe is moved down, leading to a linear relation between the buoyancy force and the indentation depth $\delta$. We can thus correct for the buoyancy force simply by extrapolating the initial part of the force curve where the probe is only moving through the liquid and not yet in contact with the hydrogel (black line in Fig. 3).

The simplest approach to avoid the dynamic contact angle and buoyancy corrections is to utilize a submersible load cell. Raw data for a 100 g-limit submersible load cell are shown in Fig. 5 with neither of the above corrections for buoyancy and contact angle hysteresis, plotted together with the corrected data from Fig. 3. We find excellent agreement for the moduli; the obtained elastic moduli $E_{\text {submerged }}$ $=951 \mathrm{~Pa}$ and $\mathrm{E}_{\text {non-submerged }}=938 \mathrm{~Pa}$ assuming $v=0.5$, measured in the swollen state, are in good agreement with values reported elsewhere. ${ }^{29}$ Note that the shaded error bars, representing the standard deviation from 5 repeated measurements on a single position on the network, are small for data obtained with the submerged load cell, while the 


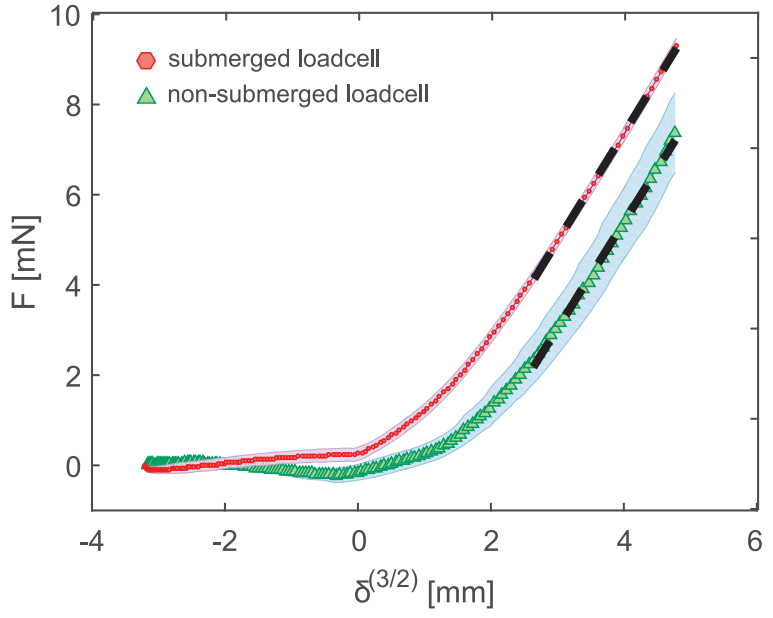

FIG. 5. Indentation of a $10 \mathrm{w} / \mathrm{v} \%$ poly(acrylamide) network crosslinked with $1.0 \mathrm{w} / \mathrm{w} \% \mathrm{~N}, N^{\prime}$-methylenebisacrylamide, measured with a non-submersible and a submersible load cell and a probe of radius $R=2 \mathrm{~mm}$. Both measurements are repeated 5 times at a single position on the network, where the first cycle corresponds to the data shown in Fig. 3. The mean of these 5 repeats are plotted along with the corresponding standard deviation, shown with shaded error bars. The dotted black lines indicate linear fits, according to Hertzian contact theory, using slopes $a_{\text {submerged }}=75.58 \mathrm{~N} / \mathrm{m}^{(3 / 2)}$ and $a_{\text {non-submerged }}=74.58 \mathrm{~N} / \mathrm{m}^{(3 / 2)}$, from which the moduli $E$ can be obtained using Eq. (1), giving $E_{\text {submerged }}=951 \mathrm{~Pa}$ and $E_{\text {non-submerged }}=938 \mathrm{~Pa}$ assuming $v=0.5$.

error bars are substantially larger for the data taken with the non-submersible load cell. Hence, even for this soft polymer network, the non-submersible load cell is more than sufficiently sensitive to accurately determine the modulus of the material.

\section{COMBINING MULTI-POSITION INDENTATION WITH FLUORESCENCE MICROSCOPY}

While the measured indentation force gives a very good estimate of the local mechanical properties of the material, much more detailed information can be obtained by simultaneously measuring the displacement field within the sample. In particular, for heterogeneous samples, which are characterized by large non-affine strains and strongly localized displacements, information on the strain field is crucial for a correct interpretation of the indentation data. Therefore, we combine our indentation setup with displacement imaging. As we will show below, the displacement maps obtained in this way can also be used to independently measure the Poisson ratio of the sample.

A common methodology to measure local displacements is by means of embedding a very low volume fraction of fluorescent tracer particles within the material. This approach requires combining fluorescence microscopy and mechanical deformation; indentation readily allows for this modification as both translation and force readout are present on the topside of the instrument, leaving the entire region below the sample available for modification. Since the displacement field in an indentation experiment will vary both in the $\hat{x}, \hat{y}$, and $\hat{z}$-directions, obtaining displacement information in all three dimensions is desirable over obtaining only two dimensional information. ${ }^{30}$ Therefore, the indentation setup is mounted on a confocal microscope, as shown in Fig. 6(a).

To measure the 3D displacement field in a hydrogel, a polyacrylamide hydrogel similar in composition to the one shown in Fig. 5 but containing a low density of fluorescent polystyrene tracer particles is indented with a spherical probe. The fluorescent beads are displaced due to the indentation (a)

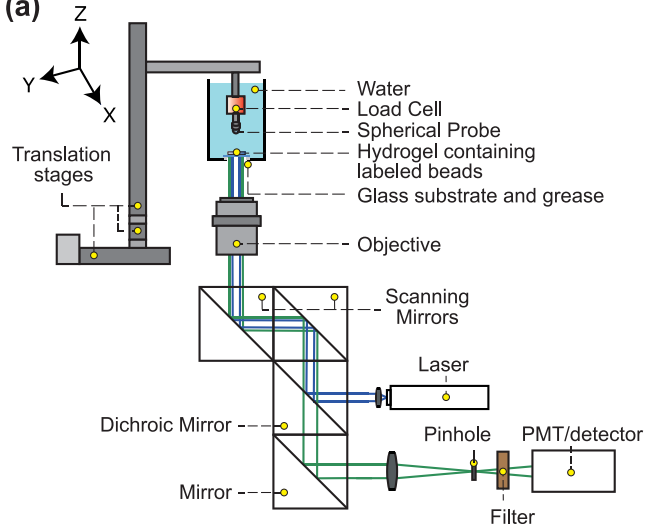

(b)

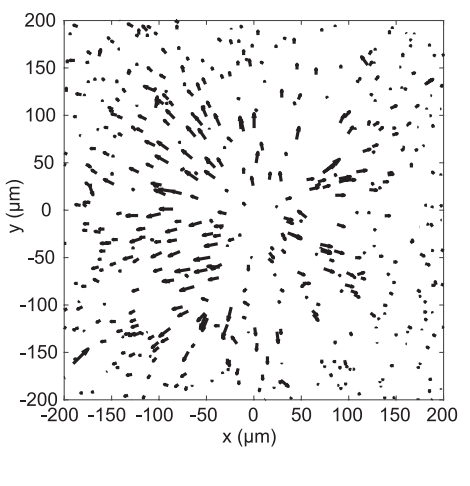

(c)

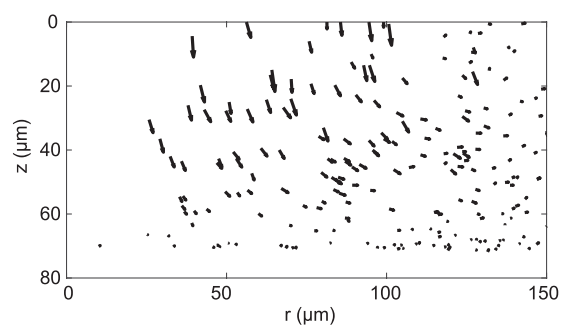

FIG. 6. (a) Schematic representation of the multi-position indentation setup combined with a Nikon C2 confocal microscope used to measure the 3D displacement field in a hydrogel. Laser light is directed to the sample using a dichroic mirror and scanning mirrors. The objective has a 20x magnification with a limited working distance of $1 \mathrm{~mm}$. The emitted light is red shifted by the fluorescent microspheres and passes the dichroic mirror and a long pass emission filter. (b) The ( $\hat{x}-\hat{y})$ projected 3D displacement field of fluorescently labeled microspheres with a diameter of $1.3 \mu \mathrm{m}$ in a hydrogel, which move due to an applied indentation with a stainless steel sphere of $500 \mu \mathrm{m}$ in diameter; radial symmetry indicates the indentation location. The arrows show the displacement over which a bead is tracked. (c) The (î-z) projected 3D displacement of the same data as in (b). 
with the spherical probe; this motion is analyzed using a locating and tracking algorithm for Matlab. ${ }^{31}$ The resulting displacement field is radially symmetric [Fig. 6(b)] and expands as the indentation depth $\delta$ increases. Making use of this radial symmetry, we plot the 3D displacement of the beads in the $(\hat{\mathrm{r}}-\hat{\mathrm{z}})$ plane in Fig. $6(\mathrm{c})$. Clearly, there is not only a displacement component in the $\hat{z}$-direction but deeper in the sample there is also a component in the $\hat{r}$ direction, which points outward. By comparing the measured displacement field with numerically calculated displacement fields for different values of $v$, we can estimate the Poisson ratio of the polyacrylamide hydrogel. In particular, the absence of an inward displacement component indicates that the Poisson ratio of the hydrogel is close to 0.5 , which is in good agreement with known values for hydrogels. ${ }^{32}$ Indeed, we find a very good agreement between the experimental and theoretical displacement maps for $v \approx 0.5$ (see the supplementary material, specifically Fig. 2S). We thus conclude that the combination of force measurements and displacement mapping allows for an independent measurement of Young's modulus and Poisson ratio.

\section{MULTI-POSITION INDENTATION OF A SPATIALLY PATTERNABLE HETEROGENEOUS POLYMER NETWORK}

The true power of the multi-position indentation instrument is exemplified by obtaining a mechanical 2D-map of a tunable mechanically heterogeneous polymer network at the millimeter scale. A test material is fabricated composed of a commercially available (methacryloxypropyl)methylsiloxanedimethylsiloxane copolymer (later on referred to as PDMS) purchased from Gelest in combination with $0.01 \mathrm{w} / \mathrm{v} \%$ of a free radical photo-initiator (2,2-dimethyl-2phenylacetophenone), resulting in a photopatternable polymer network. ${ }^{33}$ The material is halftone printed by exposing to a spatially varying intensity of UV light resulting in a spatially varying modulus. ${ }^{13}$ To obtain a flat sample, the photopatternable PDMS is sandwiched between two glass slides separated by a $127 \mu \mathrm{m}$ thick Mylar spacer; see Fig. 7(a). The glass slides are coated with a sacrificial dextran layer, obtained by spincoating a $5 \mathrm{w} / \mathrm{v} \%$ solution of $450-640 \mathrm{kDa}$ dextran in water at $2000 \mathrm{rpm}$ for $12 \mathrm{~s}$. The sacrificial layer is used to detach the polymer film from the glass substrate after UV exposure, a process that takes about 10 days soaking the sample in water and shaking on a lab shaker. Samples are exposed to UV light in a programmed pattern; ${ }^{34}$ see Fig. 7(b); the intensity of UV dosage defines the cross-linking density and therefore the local modulus of the PDMS. Unexposed PDMS is rinsed away with propylene glycol monomethyl ether acetate (PGMEA). An important note is that the spatial resolution of UV exposure $(2 \mu \mathrm{m})$ is above the polymer mesh size $\zeta$, which is estimated from the magnitude of the PDMS modulus to be on the order of $1-10 \mathrm{~nm},{ }^{35}$ using $\zeta \approx\left(\frac{E}{3 k_{B} T}\right)^{-1 / 3} \approx 1-10 \mathrm{~nm}$. As a result, this resolution is insufficient to control cross-linking at the single polymer length scale. However, naturally heterogeneous materials, such as collagen bundles or physiological tissue, are typically heterogeneous on the same length scale or larger, 1$7 \mu \mathrm{m}$ for collagen, ${ }^{36}$ than the spatial resolution of patternable PDMS.6,7

The multi-position indentation method is indeed able to map the mechanical landscape of the PDMS, corresponding to the applied dosage pattern, showing the photo-patternability of the PDMS in combination with the photo-initiator, as shown in Fig. 7(c). The local modulus is obtained from Eq. (1) with an assumed Poisson's ratio, $v=0.5$. The height of the PDMS sample is also determined by taking the difference between a zero gap measurement taken next to the material, which determines the distance between the probe and the substrate, and the position where the probe contacts the PDMS sample; this height or thickness weakly depends on the UV light dosage to which the sample is exposed, as shown in Fig. 7(d).

The sharpness of the measured elasticity pattern is limited by factors originating both from the method of sample synthesis and from the indentation methodology. First, (a)

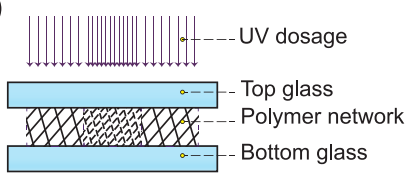

(b)

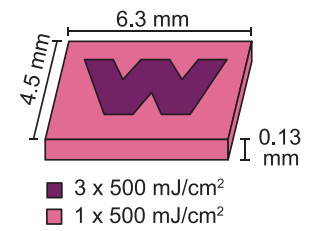

(c)

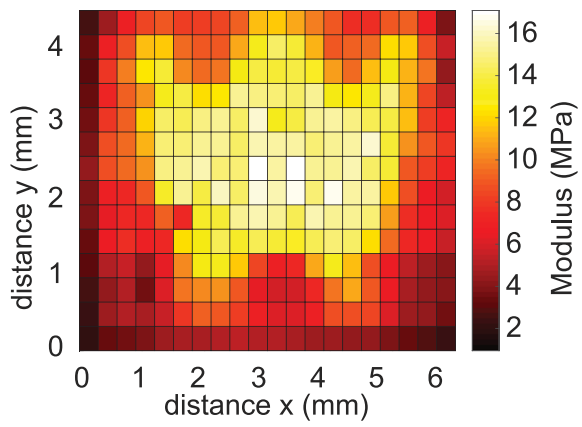

(d)

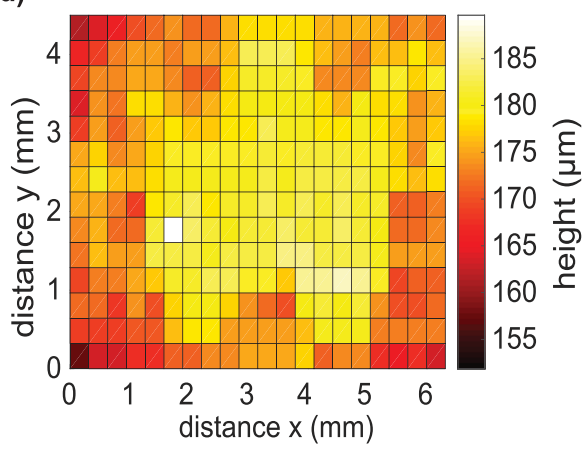

FIG. 7. (a) Spatially programmed sample preparation; the PDMS, mixed with the photo-initiator, is sandwiched between two glass slides and exposed to different dosages of UV light. This creates a proportional amount of free radicals and thereby a crosslink-gradient across the material, visualized with hatching density. (b) The spatial exposure of UV light dosage. (c) The multi-position indentation instrument is used to obtain a mechanical map of the material. The used probe has a radius $\mathrm{R}$ of $0.5 \mathrm{~mm}$. A force-threshold of $25 \mathrm{mN}$ is used to set the indentation depth $\delta$ of the probe into the material. (d) The height of the PDMS sample is measured by the multi-position indentation instrument. 
photo-initiators illuminated with UV light degrade, yielding free radicals, which crosslink the pendant methacrylate groups of the PDMS polymers. Diffusion of these free radicals will result in blurring of the features; this effect is limited by the relatively high viscosity of the PDMS. Second, broadening of UV light during exposure by both the top glass and the PDMS layer will blur the exposed features; this is reduced by producing samples that are relatively thin with respect to the exposed area; see Fig. 7(b). Third, the measured pattern is blurred by the finite size of the probe. The contact radius $a$ of the probe on the substrate depends on the probe size and the indentation depth as $a \approx \sqrt{\mathrm{R} \delta} \approx 100 \mu \mathrm{m}$, and the indenter probes the mechanical properties within a region of approximately (twice) this size. ${ }^{37}$ A smaller probe size and indentation depth will thus lead to a better spatial resolution. However, this also reduces the measured normal force which will decrease the sensitivity of the measurement, especially for very soft samples.

\section{CONCLUSION AND OUTLOOK}

In this work, we have shown that multi-position indentation is a powerful technique to spatially map the elastic modulus of mechanically heterogeneous materials over length scales larger than conventional AFM. In addition, we have shown that the indentation instrument can (i) be used in combination with experiments using fluorescent imaging, a promising combination for future experiments to, for example, accurately determine Poisson's ratio, and (ii) map samples that are submerged in a solvent. Future challenges to improve the mapping of the elastic modulus involve mechanical deconvolution of the stress field in heterogeneous materials, an area we intend to explore further using finite element simulations. In addition, the sharpness of the patterned heterogeneity can be improved by incorporating a chain-transfer agent that quenches the free radical, preventing cross-linking of the material in unexposed regions. This ability to spatially program the modulus of a polymeric material and map the resulting modulus will allow for a systematic investigation into the role of mechanical heterogeneity on the mechanical performance of materials, such as their toughness and resistance to fracture nucleation.

\section{SUPPLEMENTARY MATERIAL}

See supplementary material for a complete description and results of the numerical finite element results for determining Poisson's ratio.

\section{REFERENCES}

${ }^{1} \mathrm{M}$. Sahimi, "Non-linear and non-local transport processes in heterogeneous media: From long-range correlated percolation to fracture and materials breakdown," Phys. Rep. 306, 213-395 (1998).

2I. Yazici and O. Okay, "Spatial inhomogeneity in poly (acrylic acid) hydrogels," Polymer 46, 2595-2602 (2005).

${ }^{3} \mathrm{~S}$. Vega, M. Kwon, and J. Burdick, "Recent advances in hydrogels for cartilage tissue engineering," Eur. Cells Mater. 33, 59-75 (2017).
${ }^{4}$ F. Di Lorenzo and S. Seiffert, "Nanostructural heterogeneity in polymer networks and gels," Polym. Chem. 6, 5515-5528 (2015).

${ }^{5} \mathrm{H}$. Souguir and T. Baumberger, "Inhomogeneity development during slow, irreversible cross-linking of gelatin," in Macromolecular Symposia (Wiley Online Library, 2013), Vol. 329, pp. 41-48.

${ }^{6}$ E. M. Darling, R. E. Wilsz, M. P. Bolognesi, S. Zauscher, and F. Guilak, "Spatial mapping of the biomechanical properties of the pericellular matrix of articular cartilage measured in situ via atomic force microscopy," Biophys. J. 98, 2848-2856 (2010).

${ }^{7}$ F. Khatyr, C. Imberdis, P. Vescovo, D. Varchon, and J. Lagarde, "Model of the viscoelastic behaviour of skin in vivo and study of anisotropy," Skin Res. Technol. 10, 96-103 (2004).

${ }^{8}$ M. Bai, A. R. Missel, W. S. Klug, and A. J. Levine, "The mechanics and affinenonaffine transition in polydisperse semiflexible networks," Soft Matter 7, 907-914 (2011)

${ }^{9}$ Y. Mulla, G. Oliveri, J. T. B. Overvelde, and G. H. Koenderink, "Macroscopic and microscopic elasticity of heterogeneous polymer gels," Phys. Rev. Lett. 120, 268002 (2018).

${ }^{10}$ A. Shekhawat, S. Zapperi, and J. P. Sethna, "From damage percolation to crack nucleation through finite size criticality," Phys. Rev. Lett. 110, 185505 (2013).

${ }^{11}$ J. T. Overvelde, J. C. Weaver, C. Hoberman, and K. Bertoldi, "Rational design of reconfigurable prismatic architected materials," Nature 541, 347 (2017).

${ }^{12}$ N. Gaspar, C. Smith, and K. Evans, "Auxetic behaviour and anisotropic heterogeneity," Acta Mater. 57, 875-880 (2009).

${ }^{13}$ J. Kim, J. A. Hanna, M. Byun, C. D. Santangelo, and R. C. Hayward, "Designing responsive buckled surfaces by halftone gel lithography," Science $\mathbf{3 3 5}$, 1201-1205 (2012).

${ }^{14}$ D. H. Gracias, "Stimuli responsive self-folding using thin polymer films," Curr. Opin. Chem. Eng. 2, 112-119 (2013).

${ }^{15}$ M. Jamal, A. M. Zarafshar, and D. H. Gracias, "Differentially photocrosslinked polymers enable self-assembling microfluidics," Nat. Commun. 2, 527 (2011).

${ }^{16}$ L.-H. Cai, T. E. Kodger, R. E. Guerra, A. F. Pegoraro, M. Rubinstein, and D. A. Weitz, "Soft poly (dimethylsiloxane) elastomers from architecturedriven entanglement free design," Adv. Mater. 27, 5132-5140 (2015).

${ }^{17}$ G. Swaminathan and K. Shivakumar, "A re-examination of dma testing of polymer matrix composites," J. Reinf. Plast. Compos. 28, 979-994 (2009).

${ }^{18}$ M. E. Dokukin and I. Sokolov, "Quantitative mapping of the elastic modulus of soft materials with HarmoniX and PeakForce QNM AFM modes," Langmuir 28(46), 16060-16071 (2012).

${ }^{19}$ J. Ralston, I. Larson, M. W. Rutland, A. A. Feiler, and J. M. Kleijn, "Atomic force microscopy and direct surface force measurements," Pure Appl. Chem. 77, 2149-2170 (2005).

${ }^{20}$ E. Rettler, S. Hoeppener, B. W. Sigusch, and U. S. Schubert, "Mapping the mechanical properties of biomaterials on different length scales: Depthsensing indentation and AFM based nanoindentation," J. Mater. Chem. B $\mathbf{1}$ 2789-2806 (2013).

${ }^{21}$ Y. Hu, X. Zhao, J. J. Vlassak, and Z. Suo, "Using indentation to characterize the poroelasticity of gels," Appl. Phys. Lett. 96, 121904 (2010).

${ }^{22}$ V. L. Popov, Contact Mechanics and Friction (Springer, 2010).

${ }^{23}$ E. P. Chan, Y. Hu, P. M. Johnson, Z. Suo, and C. M. Stafford, "Spherical indentation testing of poroelastic relaxations in thin hydrogel layers," Soft Matter 8, 1492-1498 (2012).

${ }^{24}$ MATLAB, version R2016b, The MathWorks, Inc., Natick, Massachusetts, 2016.

${ }^{25}$ A. S. Hoffman, "Hydrogels for biomedical applications," Adv. Drug Delivery Rev. 64, 18-23 (2012).

${ }^{26} \mathrm{~A}$. Chakrabarti and M. K. Chaudhury, "Direct measurement of the surface tension of a soft elastic hydrogel: Exploration of elastocapillary instability in adhesion," Langmuir 29, 6926-6935 (2013). 
${ }^{27}$ B. He, J. Lee, and N. A. Patankar, "Contact angle hysteresis on rough hydrophobic surfaces," Colloids Surf., A 248, 101-104 (2004).

${ }^{28}$ D. C. Giancoli, Physics for Scientists and Engineers (Pearson Education International, 2008).

${ }^{29}$ A. K. Denisin and B. L. Pruitt, "Tuning the range of polyacrylamide gel stiffness for mechanobiology applications," ACS Appl. Mater. Interfaces 8 , 21893-21902 (2016)

${ }^{30}$ M. A. J. Cox, J. B. Driessen, R. A. Boerboom, C. V. C. Bouten, and F. P. T. Baaijens, "Mechanical characterization of anisotropic planar biological soft tissues using finite indentation: Experimental feasibility," J. Biomech. 41, 422-429 (2008).

${ }^{31}$ Y. Gao and M. L. Kilfoil, "Accurate detection and complete tracking of large populations of features in three dimensions," Opt. Express 17, 4685-4704 (2009).
${ }^{32}$ E. Geissler and A. M. Hecht, "The Poisson ratio in polymer gels," Macromolecules 14, 185-188 (1981)

${ }^{33}$ K. M. Choi and J. A. Rogers, "A photocurable poly (dimethylsiloxane) chemistry designed for soft lithographic molding and printing in the nanometer regime," J. Am. Chem. Soc. 125, 4060-4061 (2003).

${ }^{34}$ Durham Magneto Optics Ltd., MicroWriter ML3 Baby, Durham DH1 3HP, UK.

${ }^{35}$ F. Di Lorenzo, J. Hellwig, R. Von Klitzing, and S. Seiffert, "Macroscopic and microscopic elasticity of heterogeneous polymer gels," ACS Macro Lett. 4, 698-703 (2015).

${ }^{36}$ I. K. Piechocka, A. S. G. Oosten, R. G. M. Breuls, and G. H. Koenderink, "Rheology of heterotypic collagen networks," Biomacromolecules $\mathbf{1 2}$ 2797-2805 (2011) 